\title{
Enterovirus D68 and disease severity: more questions than answers
}

\author{
Michelle Science MD MSc, Upton Allen MBBS MSc
}

See also www.cmaj.ca/lookup/doi/10.1503/cmaj.150619

I n North America, nonpolio enteroviruses cause millions of infections each year. ${ }^{1}$ These RNA viruses include more than 100 serotypes that are grouped into 4 species (A, B, $\mathrm{C}$ and D). Enterovirus D68 (EV-D68) is a specific strain that is known to be associated with respiratory illness. It was first identified in 1962, and clusters - mainly affecting children - have been infrequently described. ${ }^{2}$ During the 2014 outbreak in North America, severe cases requiring admission to an intensive care unit were reported. In addition, the association between EV-D68 and neurologic illness, including acute flaccid paralysis, was reported.

Before the 2014 outbreak, it was known that certain groups of patients were at risk of severe enterovirus disease. These groups included newborns, patients with humoral or combined immune deficiencies, recipients of stem cell or solid organ transplants, children with malignant disease and patients receiving anti-CD20 monoclonal antibodies. ${ }^{3}$ In the 2014 outbreak, severe cases were predominantly found among children with underlying asthma or a history of wheezing. ${ }^{4,5}$ Obesity was also proposed as a risk factor. ${ }^{6}$ These observations, along with reports of neurologic illness, prompted substantial public interest in this new "severe" pathogen. However, beyond case reports and case series, few studies have either assessed whether EV-D68 truly causes more severe respiratory disease compared with other pathogens or identified risk factors for respiratory disease using appropriate scientific methods.

In the accompanying article, ${ }^{7}$ Mertz and colleagues compared the characteristics and outcomes of patients with EV-D68 with those with non-EV-D68 rhinovirus or enterovirus. This matched-cohort study adds to the literature by providing a direct comparison between EV-D68 and other rhinoviruses or enteroviruses. They concluded that EV-D68 appears to be a more virulent pathogen based on patients' increased difficulty with breathing at presentation, increased odds of admission and increased need for magnesium sulfate and intravenous adminis- tration of salbutamol. When these outcomes were adjusted for history of allergy and/or other comorbidities, the odds of admission and need for salbutamol were no longer statistically significant, and no differences in the rate of admission to the pediatric critical care unit were seen. In addition, there were no differences in mortality or in the need for invasive or noninvasive mechanical ventilation. The study did not examine underlying asthma severity as a potential confounder. In this regard, it would be important to ensure that the study groups were comparable with respect to the proportion of patients with severe asthma in each group - patients with severe asthma are more likely to be admitted to hospital and receive the aforementioned interventions regardless of their EV-D68 status.

This minor consideration notwithstanding, similar findings were documented in a recent study by Schuster and colleagues comparing patients with EV-D68 and non-EV-D68 enterovirus or rhinovirus admitted to a pediatric critical care unit, where children with EV-D68 were more likely to require magnesium, albuterol and aminophylline. ${ }^{8}$

A study from Alberta compared patients with EV-D68 to those with other enterovirus strains to determine whether the former group of patients was more likely to have respiratory illness. ${ }^{9}$ Although the researchers found an association between asthma and EV-D68, the results were based on information from administrative and surveillance databases, and therefore the full clinical spectrum on disease severity and outcomes was not available. In the study by Mertz and colleagues, patients with EV-D68 were more likely to

\section{- KeY POINTS}

- An outbreak of enterovirus D68 associated with respiratory illness occurred in 2014.

- Possible risk factors for severe disease include history of atopy or asthma.

- Whether EV-D68 is truly more severe than other respiratory viruses remains to be determined.

- Enteroviruses can causes a range of symptoms, but association with neurologic complications is unproven. 
have a family history of allergy, and a trend toward increased personal atopy that did not reach statistical significance was seen. Schuster and colleagues did document statistically significant increased odds of asthma or recurrent wheeze in patients admitted to intesive care with EV-D68. ${ }^{8}$ Therefore, although it is important to consider that history would have been dependent on parental self-report and provider documentation, it seems reasonable to hypothesize that EV-D68 may be a more virulent pathogen in patients with preexisting atopic disease when compared with rhinoviruses and other enteroviruses.

Why were infants, children and teenagers predominantly affected in the EV-D68 outbreak? The likely explanation is that people in these age groups do not yet have immunity from previous exposure to these viruses. Illness is therefore more likely to develop after exposure in these patients than in their adult counterparts. With respect to the association between EV-D68 and asthma or atopy, questions arise relating to the immunologic basis of this association. In this regard, a possible explanation might lie in the relative balance of $T$ helper cell $\left(T_{h}\right) 1$ to $T_{h} 2$ in the patient, with the $T_{h} 2$ profile more likely to be associated with asthma and atopy. $\mathrm{A}_{\mathrm{h}} 2$ profile is associated with less cell-mediated immune control and, theoretically, an increased susceptibility to infections in which cell-mediated immunity plays a role in controlling, notwithstanding the pivotal role that humoral immunity has in the defense against enteroviral infections..$^{10}$

Whether there are viral genetic factors that contributed to the 2014 outbreak is a question worthy of further study. Although EV-D68 is a member of the enterovirus D species, it has phenotypic characteristics that are more consistent with rhinoviruses. Whether the strains that were associated with the 2014 outbreak are characterized by special virulence factors that contribute to illness severity remains to be addressed.

Although beyond the scope of the accompanying article, the potential role of EV-D68 in neurologic complications deserves specific mention. Enteroviruses are known to be associated with neurologic manifestations including meningitis, encephalitis and acute flaccid paralysis. Shortly after EV-D68 was noted to be causing respiratory illness, an apparent increase in cases of acute flaccid paralysis was seen, which subsequently declined with the decrease in EV-D68 respiratory disease. This temporal relationship raised the question as to whether EV-D68 could be responsible for the cases of acute flaccid paralysis. The virus was found in respiratory specimens from some, but not all, children with acute flaccid paralysis. ${ }^{11}$ Thus, the role of EV-D68 in acute flaccid paralysis associated with the 2014 outbreak is yet to be determined. Indeed, some evidence suggests that enteroviruses other than EV-D68 may have contributed to the acute flaccid paralysis. ${ }^{12}$

Further studies are needed to define whether EV-D68 is truly a more severe pathogen than other enteroviruses and rhinoviruses for all patients, or whether there are certain populations at increased risk of severe disease. Such research should also address whether there are factors that are related to viral or patient genetic variation that might be associated with disease severity.

\section{References}

1. Non-polio enterovrius. Atlanta (GA): Centers for Disease Control and Prevention. Available: www.cdc.gov/non-polio-enterovirus/ about/overview.html (accessed 2015 May 5).

2. Clusters of acute respiratory illness associated with human enterovirus 68: Asia, Europe, and United States, 2008-2010. MMWR Morb Mortal Wkly Rep 2011;60:1301-4.

3. American Academy of Pediatrics (Enteroviruse -nonpoliovirus) In: Kimberlin DW, Brady MT, Jackson MA, editors. Red Book: 2015 Report of the Committee on Infectious Diseases. 30th ed. Elk Grove Village (IL): American Academy of Pediatrics; 2015: 333-6.

4. Midgley CM, Jackson MA, Selvarangan R, et al. Severe respiratory illness associated with enterovirus D68: Missouri and Illinois, 2014. MMWR Morb Mortal Wkly Rep 2014;63:798-9.

5. Enterovirus D68. Atlanta: Centers for Disease Control; 2015. Available: www.cdc.gov/non-polio-enterovirus/about/ev-d68. html (accessed 2015 May 6).

6. Farrell JJ, Ikladios O, Wylie KM, et al. Enterovirus D68-associated acute respiratory distress syndrome in adult, United States, 2014. Emerg Infect Dis 2015;21:914-6.

7. Mertz D, Alawfi A, Pernica JM, et al. Clinical severity of pediatric respiratory illness with enterovirus D68 compared with rhinovirus or other enterovirus genotypes. CMAJ 2015 Oct. 13 [Epub ahead of print].

8. Schuster JE, Miller JO, Selvarangan R, et al. Severe enterovirus 68 respiratory illness in children requiring intensive care management. J Clin Virol 2015;70:77-82.

9. Drews SJ, Simmonds K, Usman HR, et al. Characterization of enterovirus activity, including that of enterovirus D68, in pediatric patients in Alberta, Canada, in 2014. J Clin Microbiol 2015;53: 1042-5.

10. Chang L-Y, Hsiung CA, Lu C-Y, et al. Status of cellular rather than humoral immunity is correlated with clinical outcome of enterovirus 71. Pediatr Res 2006;60:466-71.

11. Greninger AL, Naccache SN, Messacar K, et al. A novel outbreak enterovirus D68 strain associated with acute flaccid myelitis cases in the USA (2012-14): a retrospective cohort study. Lancet Infect Dis 2015;15:671-82.

12. Horner LM, Poulter MD, Brenton JN, et al. Acute flaccid paralysis associated with novel enterovirus C105. Emerg Infect Dis 2015;21: 10.3201/eid2110.150759 (accessed 2015 Sept. 14).

Affiliations: Division of Infectious Diseases (Science, Allen), Department of Paediatrics; Children Health Evaluative Sciences (Allen), Research Institute, The Hospital for Sick Children, University of Toronto, Toronto, Ont.

Contributors: The authors contributed equally to the initial draft and revisions of the manuscript. Both of the authors approved the version to be published and agree to act as guarantors of the work. 